\title{
AVALIAÇÃO ECONÔMICA FINANCEIRA DE INVESTIMENTO EM TROCA DE MISTURADOR PARA INDÚSTRIA DE RAÇÃO ANIMAL
}

\section{ECONOMIC AND FINANCIAL EVALUATION OF INVESTMENT IN EXCHANGE OF BEATER FOR ANIMAL FEED INDUSTRY}

${ }^{1}$ Felipe Kesrouani Lemos, ${ }^{2}$ Aline Marques Fernandes dos Santos, ${ }^{2}$ Gabriel Simões, ${ }^{2}$ Renato Henrique Niz da Silva.

1 Universidade do Oeste Paulista - UNOESTE, Curso de Engenharia de Produção, Presidente Prudente, SP. e-mail: felipeklemos@unoeste.br

RESUMO - O objetivo do presente trabalho é explorar a viabilidade econômico-financeira do investimento em uma reposição de ativo no setor de ração animal. $O$ estudo não apenas se baseou na modelagem do fluxo de caixa estimado, mas também na avaliação de seu risco, utilizando uma abordagem probabilística, valendo-se da técnica de simulação de Monte Carlo. O modelo foi calibrado fazendo testes iniciais para balancear esforço computacional e estabilidade dos resultados. Os resultados foram marcados por uma dispersão muito grande de resultados, o que mostra o risco existente no setor. Apesar do resultado favorável em média, o alto desvio-padrão mostrou um comportamento próximo do aleatório em uma ampla faixa de valores. Recomenda-se, portanto, estratégias de risco para investimentos como o apresentado, como a buscar por travas financeiras da matéria-prima. Palavras-chave: Simulação de Monte Carlo. Análise de Investimento. Indústria de ração animal.

ABSTRACT - The aim of this work is to explore both economic and financial viability of investing in asset replacement for the animal feeding sector. The study is not only based on the modeling of the flow the estimated cash, but also in the evaluation of its risk through a probabilistic approach, using Monte Carlo simulation technique. The model was calibrated by the making of initial tests in order to balance computational effort with stability of results. The results were greatly dispersed, which shows the existing risk at the sector. Despite of the average favorable result, the high standard deviation showed a nearly random behavior in a wide range of values. Therefore, strategies of risk are recommended for investments like the one presented, such as the searching for financial stops of the raw material.

Keywords: Simulation Monte Carlo. Investment Analysis. Feed industry. 


\section{INTRODUÇÃO}

A crescente demanda do mercado mundial por carne bovina de qualidade, proveniente de animais criados a pasto, gera ótimas perspectivas para a pecuária brasileira. Isso exige dos produtores excelência em três principais fatores: nutrição correta; controle sanitário eficiente e melhoramento genético.

A alimentação representa cerca de 70 a $80 \%$ dos custos de produção animal, e é base fundamental para um bom manejo desses animais futuramente, há cada vez mais a necessidade de se ter uma boa alimentação para se ter um bom produto final. Como o terceiro maior produtor de ração animal, o Brasil apresenta um crescimento do setor de aproximadamente $4 \%$, em 2012, produzindo mais de 58,4 milhões de toneladas de janeiro a novembro de 2013 (FACHINI, 2014).

O processo de fabricação de ração animal conta com modelos de programação linear de mínimo custo para resolver problemas nas formulações de rações animais. Porém, depende necessariamente de misturadores capazes de realizar uma boa homogeneização, com o objetivo de garantir que todos os animais consumam a ração nas mesmas proporções.

A importância de se trabalhar com misturadores traz para a indústria de ração agilidade e melhor qualidade no produto.
Fatores como a mistura através de giros do maquinário, posição de trabalho e tempo de preparo devem ser levados em consideração, pois uma má funcionalidade nesses sistemas pode acarretar em deficiências no ganho de peso e produtividade dos animais.

Segundo Pessoa (2003) a falha de mistura causada por equipamentos é capaz de afetar diretamente a posição de mercado da empresa, não trazendo benefícios para tais, por isso, é necessário que haja treinamentos específicos para os funcionários na forma de se manusear e as exigências necessárias para 0 bom funcionamento.

Para suprir o crescimento do setor e assegurar a qualidade do produto final as empresas do ramo estão buscando investir em novos maquinários, mais tecnológicos e com maior eficiência.

Portanto, para projetar o sucesso ou insucesso de um investimento é possível basear-se em uma simulação. A simulação, de acordo com Shamblin (apud OLIVEIRA, 2008), é uma ferramenta muito valiosa por permitir obter uma resposta a um problema particular. Ou seja, a partir da simulação pode-se imitar um processo real, gerando uma história e dados artificiais e através do estudo desses dados fazer uma estimativa de resultados do projeto.

Através dessas análises os gestores podem avaliar a viabilidade de um 
investimento para alavancar sua produção e gerar maior competitividade no mercado.

O seguinte trabalho tem como objetivo demonstrar a viabilidade na troca de misturadores de uma fábrica onde se produz ração animal por misturadores de um melhor desempenho, sendo mais novos e de melhor funcionalidade para a empresa, utilizando critérios da Engenharia Econômica que permitam elucidar os mecanismos para otimização de recursos aplicados em projetos de engenharia.

\section{METODOLOGIA}

0 presente trabalho foi desenvolvido a partir de uma simulação de troca de maquinário de uma empresa de ração animal. Foram coletados alguns dados reais sobre um misturador, e outra parte dos dados foi estipulado.

\subsection{Coleta de Dados}

Os dados coletados foram obtidos através de entrevista com profissionais experientes na área. Estes estão descritos na tabela abaixo:

Tabela 1. Dados coletados em entrevistas para o modelo

\begin{tabular}{|c|c|c|c|}
\hline \multicolumn{4}{|c|}{ Dados } \\
\hline Preço do misturador & RS & $180.000,00$ & RS \\
\hline Tempo de depreciação & & 10 & anos \\
\hline Valor residual & & $40 \%$ & $\%$ equipamento \\
\hline Velocidade & & 20 & thr \\
\hline Potencial a produzir & & 42240 & tano \\
\hline Produfvidade - Sal & & $70 \%$ & t'mês \\
\hline Produfvidade - Ração & & $30 \%$ & t'mês \\
\hline Preço da sal & & 33 & $\mathrm{R} \$ / \mathrm{sc}$ \\
\hline Preço da Ração & & 28 & $\mathrm{R} \$ / s C$ \\
\hline Percentual do tempo misturando & & $50 \%$ & unidades \\
\hline Custo produzir sal & & 29,5 & $\mathrm{R} \$ / \mathrm{sC}$ \\
\hline Custo produzir Ração & & 25,5 & $\mathrm{RS} / \mathrm{sc}$ \\
\hline Manutenção & & $1,00 \%$ & $\%$ do equipamento \\
\hline Mão-de-obra & RS & $3.300,00$ & RS/salário \\
\hline
\end{tabular}

Os impostos e contribuições baseados nos valores encontrados na Receita federais utilizados no presente trabalho são Federal do Brasil. 
Tabela 2. Carga tributária considerada

\begin{tabular}{l|c|c} 
PIS & $3 \%$ & \% da recetta \\
COFINS & $0,65 \%$ & \% da receita \\
IPI & $5 \%$ & \% da recetta \\
IRPJ & $25 \%$ & \% do lucro \\
CSLL & $9 \%$ & \% do lucro \\
Encargos sociais & $20 \%$ & \% da folha \\
Taxa de atrasvidade & $10 \%$ & \% a.a.
\end{tabular}

\subsection{Modelo}

A primeira etapa da simulação é a construção da $\mathrm{DRE}^{1}$ baseada nas informações coletadas, onde foram calculados os seguintes dados:

\footnotetext{
${ }^{1}$ DRE (Demonstração do Resultado do Exercício) é uma demonstração contabilística dinâmica que se destina a evidenciar a formação do resultado líquido em um exercício, através do confronto das receitas, custos e resultados, apuradas segundo o princípio contábil do regime de competência.
} 


$$
\begin{gathered}
R B=\text { Ppro } \times\left(\left(\text { Prodsal } \times \text { psal } \times \frac{1000}{30}\right)+\left(\text { Prodraç } \times \text { praç } \times \frac{1000}{30}\right)\right) \times \text { PrecMis } \\
I S R=\text { PrecMis } \times(\text { PIS }+ \text { COFINS }+ \text { IPI }) \\
R L=R B-I S R
\end{gathered}
$$$$
\text { Cprods }=P P \times P e r M a n \times \text { Prodsal } \times \text { Cs } \times\left(\frac{1000}{30}\right)
$$$$
\text { Cprodr }=P P \times P e r M a n \times P r o d r a c ̧ \times C r \times\left(\frac{1000}{30}\right)
$$$$
\text { Man }=\text { PrecMis } \times \text { PerMan } \times 12
$$$$
C=\text { Cprods }+ \text { Man }+ \text { Cprodr }
$$$$
M O=M O \times 13,33 \times(1+E S)
$$$$
L A J I D A=R L-C
$$$$
D e=\frac{\text { PrecMis }}{\text { Td }}
$$$$
L A I R=L A J I D A-D e
$$$$
I R=L A I R \times(I R P J+C S L L)
$$$$
L C=L A I R-I R
$$$$
F o=L C+D e
$$$$
\text { Fo0 }=- \text { PrecMis }
$$$$
F o 1=F o
$$$$
F o 2=F o
$$$$
\mathrm{Fo3}=\mathrm{Fo}
$$$$
\text { Fo4 }=F o
$$$$
F o 5=F o
$$$$
F o 6=F o
$$$$
F o 7=F o
$$

$F o 8=F o$
$F o 9=F o$

$$
\begin{gathered}
\text { Fo10 }=\text { Fo }+(\text { PrecMis }+ \text { Vr }) \\
\text { VPL }=\sum_{t=0}^{n}\left(\frac{\text { Fot }}{(1+i)^{t}}\right)
\end{gathered}
$$

A $T_{I R}^{2}$ foi calculada no Excel.

\footnotetext{
${ }^{2}$ TIR (Taxa Interna de Retorno) é uma taxa de desconto hipotética que, quando aplicada a um fluxo de caixa, faz com que os valores das despesas, trazidos ao valor presente, seja igual aos valores dos retornos dos investimentos, também trazidos ao valor presente.
} 
Tabela 3. DRE Projetada e Fluxo de caixa

\begin{tabular}{|c|c|c|c|c|c|}
\hline \multicolumn{3}{|c|}{ DRE projetada } & Ano & \multicolumn{2}{|r|}{$\mathrm{FCL}$} \\
\hline Receita bruta & RS & $22.176 .000,00$ & 0 & -RS & $180.000,00$ \\
\hline$(-)$ Impostos sobre receita & RS & $1.918 .224,00$ & 1 & RS & $212.684,16$ \\
\hline Receita liquida & RS & $20.257 .776,00$ & 2 & RS & $212.684,16$ \\
\hline$(-)$ Custos & RS & $19.944 .800,00$ & 3 & RS & $212.684,16$ \\
\hline Produção de sal & RS & $14.537 .600,00$ & 4 & RS & $212.684,16$ \\
\hline Manutenção & RS & $21.600,00$ & 5 & RS & $212.684,16$ \\
\hline Produção de ração & RS & $5.385 .600,00$ & 6 & RS & $212.684,16$ \\
\hline $\begin{array}{l}\text { Mao de obra } \\
\text { LAJIDA }\end{array}$ & RS & $\begin{array}{r}52.780,80 \\
312.976,00\end{array}$ & 7 & RS & $212.684,16$ \\
\hline Depreciação & RS & $18.000,00$ & 8 & RS & $212.684,16$ \\
\hline LAIR & RS & $294.976,00$ & 9 & RS & $212.684,16$ \\
\hline$(-) \mathbb{R}$ & RS & $100.291,84$ & 10 & RS & $284.684,16$ \\
\hline \multirow[t]{2}{*}{ Lucro contábil } & RS & $194.684,16$ & & & \\
\hline & & & TIR & & $118,1 \%$ \\
\hline Fluxo da operação & RS & $212.684,16$ & VPL & RS & $1.154 .611,21$ \\
\hline
\end{tabular}

Após a montagem do DRE e o Fluxo de Caixa obtido, foi realizado dois tipos de análises: Análise de Ce

A primeira análise foi realizada em

três cenários diferentes (realista, otimista e pessimista), para avaliar o comportamento dos dados e os resultados de cada situação, onde as informações de produtividade, custos de mão de obra e manutenção, valor residual e preço de venda variam para cada cenário. Já a análise de sensibilidade avalia os lucros obtidos com a variação no preço de um produto.

Por fim, realizou-se a simulação de Monte $\mathrm{Carlo}^{3}$ que apresentou os resultados abaixo.

\section{RESULTADOS E DISCUSSÃO}

\subsection{Calibração dos Parâmetros de Simulação}

Para a efetivação da simulação de Monte Carlo fez-se necessário realizar primeiro a calibração de seus parâmetros. Através dessa etapa foi possível identificar o melhor número de simulações, o seja, aquele que apresentou menor desvio padrão das médias.

Para valores estipulados de número de simulações - n, foi realizado dez simulações de $n$, onde se obteve valores distintos de médias, para os quais foi calculado um desvio padrão. As médias obtidas estão relacionadas na tabela abaixo:

\footnotetext{
${ }^{3}$ Monte Carlo é um método estatístico que se baseia em amostragens aleatórias massivas para obter resultados numéricos, isto é, repetindo sucessivas simulações um elevado número de vezes, para calcular probabilidades.
} 
Tabela 4. Calibração do número de simulações

\begin{tabular}{|c|c|c|c|c|c|c|}
\hline & \multicolumn{6}{|c|}{ Simulações } \\
\hline Médias & 10 & 50 & 100 & 200 & 500 & 1000 \\
\hline media 1 & -R\$ 257.098,11 & $\mathrm{R} \$ 161.855,31$ & $\mathrm{R} \$ \quad 51.312,40$ & R\$ $79.541,26$ & $\mathrm{R} \$ 155.278,16$ & R\$ $236.776,95$ \\
\hline media 2 & -R\$ $71.173,84$ & $\mathrm{R} \$ 357.638,63$ & $\mathrm{R} \$ 147.293,98$ & $\mathrm{R} \$ 126.481,77$ & $\mathrm{R} \$ 220.221,02$ & $\mathrm{R} \$ 209.876,52$ \\
\hline media 3 & $\mathrm{R} \$ 273.311,20$ & $\mathrm{R} \$ \quad 9.886,86$ & $\mathrm{R} \$ 312.001,03$ & R\$ $81.170,31$ & R\$ 218.369,94 & $\mathrm{R} \$ 181.552,05$ \\
\hline media 4 & -R\$ 178.997,71 & -R\$ $123.189,60$ & -R\$ $81.913,55$ & $\mathrm{R} \$ 195.702,29$ & $\mathrm{R} \$ 176.100,06$ & $\mathrm{R} \$ 181.686,96$ \\
\hline media 5 & -R\$ 205.149,11 & $\mathrm{R} \$ \quad 21.406,97$ & $\mathrm{R} \$ 323.239,61$ & $\mathrm{R} \$ 216.997,80$ & $\mathrm{R} \$ 242.712,69$ & $\mathrm{R} \$ 142.961,96$ \\
\hline media 6 & -R\$ $\quad 2.336,50$ & $\mathrm{R} \$ \quad 98.578,15$ & RS $192.919,67$ & $\mathrm{R} \$ 178.090,42$ & R\$ $215.551,03$ & $\mathrm{R} \$ 155.385,71$ \\
\hline media 7 & $\mathrm{R} \$ 115.110,92$ & $\mathrm{R} \$ 279.238,51$ & $\mathrm{R} \$ 236.773,05$ & $\mathrm{R} \$ 238.202,89$ & $\mathrm{R} \$ 294.439,15$ & $\mathrm{R} \$ 262.170,58$ \\
\hline media 8 & $\mathrm{R} \$ 182.454,47$ & $\mathrm{R} \$ \quad 91.674,17$ & $\mathrm{R} \$ 296.510,81$ & $\mathrm{R} \$ 131.245,45$ & $\mathrm{R} \$ 229.006,69$ & $\mathrm{R} \$ 183.815,20$ \\
\hline media 9 & $\mathrm{R} \$ 332.855,65$ & $\mathrm{R} \$ \quad 4.697,76$ & $\mathrm{R} \$ 192.235,04$ & $\mathrm{R} \$ 201.637,70$ & $\mathrm{R} \$ 251.067,71$ & $\mathrm{R} \$ 250.629,68$ \\
\hline media 10 & $\mathrm{R} \$ 360.332,77$ & $\mathrm{R} \$ 265.301,36$ & R\$ $277.570,24$ & $\mathrm{R} \$ 261.959,34$ & $\mathrm{R} \$ 105.951,67$ & $\mathrm{R} \$ 247.852,07$ \\
\hline desvio-padrão & $\mathrm{R} \$ 230.215,98$ & R\$ $149.118,74$ & R\$ $128.502,73$ & $\mathrm{R} \$ \quad 63.684,15$ & $\mathrm{R} \$ \quad 53.140,71$ & R\$ $42.258,54$ \\
\hline tempo (s) & 0 & 2 & 3 & 6 & 14 & 27 \\
\hline
\end{tabular}

O gráfico demonstra o ponto onde se obtêm o melhor resultado para $n$, que é o encontro do menor desvio padrão com o menor tempo gasto para calcular.

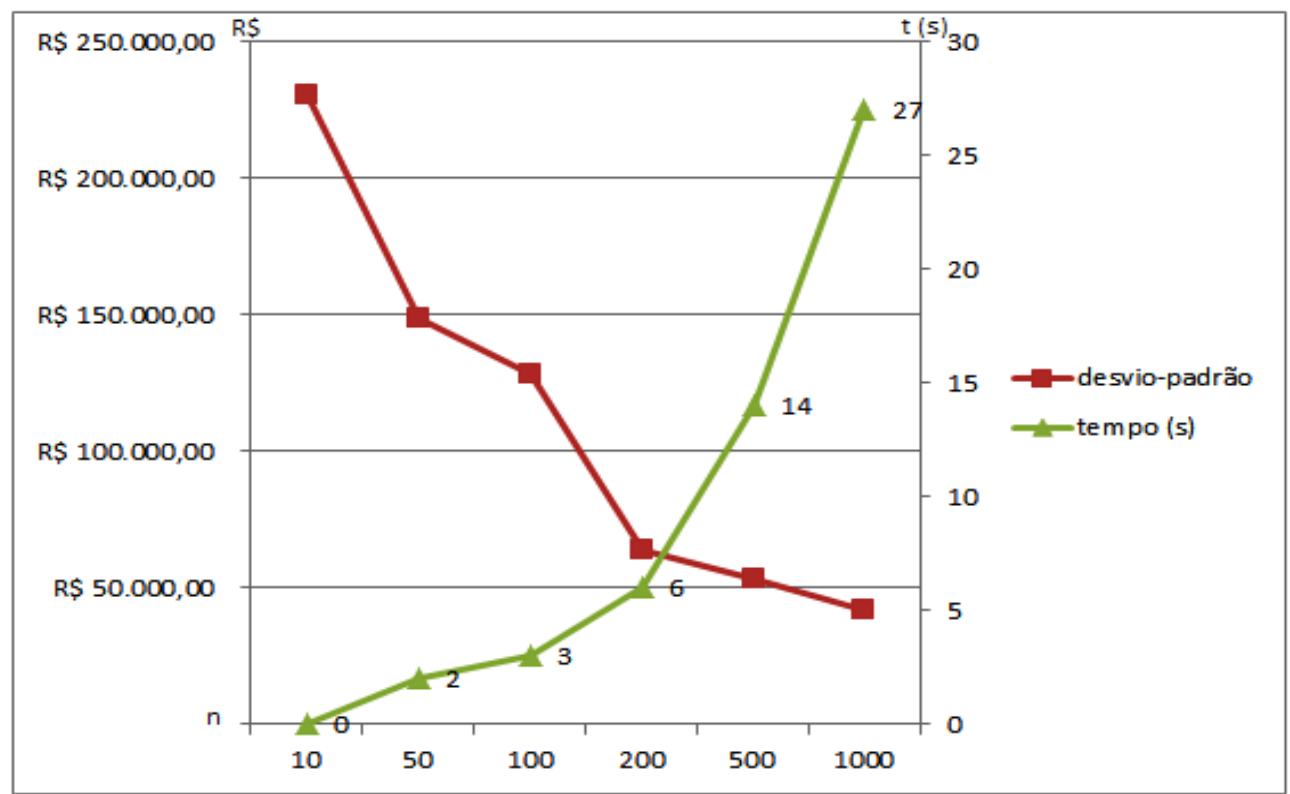

Figura 1. Gráfico da relação entre o número de simulação e o custo computacional (tempo de processamento) e estabilidade dos resultados.

Fonte: O próprio autor

\subsection{Resultados}

A análise dos dados apresentou os seguintes valores que constam na Tabela 5. 
Tabela 5. Resultados obtidos

\begin{tabular}{|c|c|}
\hline Média & RS 232.466,33 \\
\hline Desvio & RS $1.204 .806,27$ \\
\hline \% de insucessos & $45 \%$ \\
\hline Minimo & -RS $2.626 .257,76$ \\
\hline Máximo & RS $2.965 .772,18$ \\
\hline
\end{tabular}

\begin{tabular}{|c|c|}
\hline Faixa & Frequência \\
\hline Abaixo de - R\$ $2.000 .000,00$ & $0,9 \%$ \\
\hline De - R\$ 2.000.000,00 a - R\$ $1.250 .000,00$ & $12,8 \%$ \\
\hline De - R\$ $1.250 .000,00$ a - R\$ $500.000,00$ & $18,7 \%$ \\
\hline De - R\$ 500.000,00 a R\$ $250.000,00$ & $18,7 \%$ \\
\hline De R\$ $1.000 .000,00$ a R\$ $1.750 .000,00$ & $17,8 \%$ \\
\hline De R\$ $1.750 .000,00$ a R\$ $2.500 .000,00$ & $18,0 \%$ \\
\hline De R\$ 2.500.000,00 a R\$ 3.250.000,00 & $11,9 \%$ \\
\hline De R\$ 3.250.000,00 a R\$ 4.000.000,00 & $1,2 \%$ \\
\hline Acima de R\$ 4.000.000,00 & $0,0 \%$ \\
\hline
\end{tabular}

Em média, o valor presente líquido mostrou-se positivo, de $\mathrm{R} \$ 2^{232.466,33 .}$ Entretanto, chama-se atenção nos resultados para sua alta dispersão. A Figura 2 mostra a distribuição dos resultados obtidos, observando-se que trata-se de uma distribuição de base bastante alargada, assemelhando-se a uma distribuição uniforme. Como é sabido, esta é a distribuição que retrata eventos aleatórios, o que é um indicador do grande risco do negócio em questão.

Corrobora para essa observação o grande desvio padrão ( $R \$ 1,2$ milhão de reais), que é quase 6 vezes o valor da média. Resulta disto o dado de $45 \%$ de insucesso do modelo. 


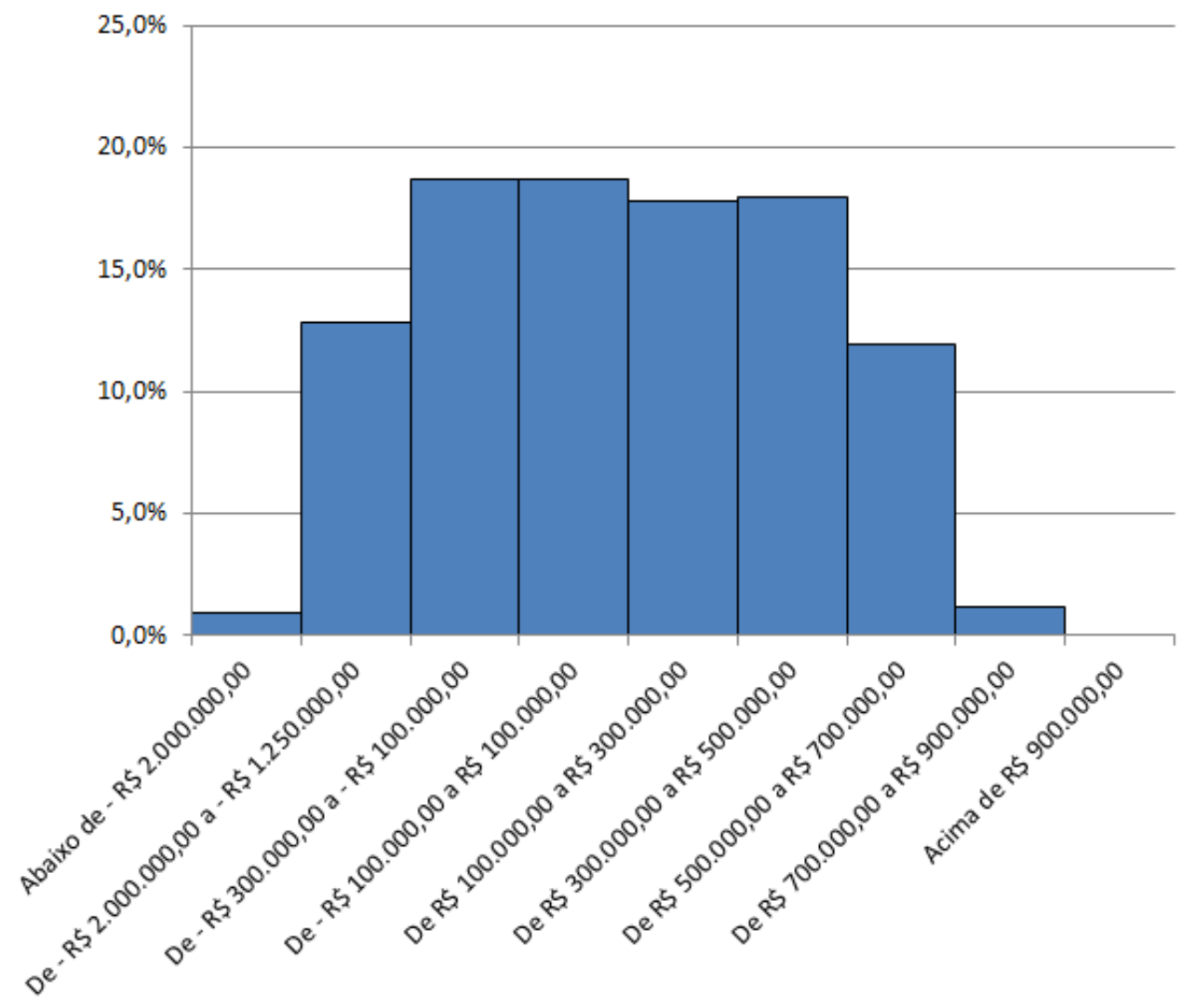

Figura 2. Distribuição do resultado final nas simulações.

Fonte: O próprio autor

\section{CONCLUSÃOS}

Os resultados obtidos mostram que o setor de ração animal insere-se em um contexto de grande risco. Notoriamente, as margens apertadas são o maior detrator dos resultados, levando a resultados negativos em certas conjunturas, potencializados pelo grande multiplicador das quantidades obtidas.

Os altos custos variáveis somam-se às incertezas de sua formação de preços, que é ditado pelo mercado de commodities. Assim, fatores ambientais interferem de sobremaneira nas margens do setor.

Este horizonte leva a uma preocupação grande do setor com o seu risco de preços. Instrumentos como o hedge de insumos na bolsa de valores são bastante indicados para não exporem a empresas a riscos de flutuações de suas principais matérias-primas.

$$
\text { Para alavancar a produção e }
$$
garantir espaço no mercado as empresas do setor de ração animal estão buscando investir em melhores máquinas, capazes de produzir mais em menor tempo. A troca dos misturadores antigos por novos necessita de um grande investimento de capital. E para uma situação como essa é preciso analisar todos os fatores que influenciam nos resultados. 


$$
\text { Este trabalho apresentou a }
$$

simulação de resultados obtidos com a troca de maquinário, e observou que, com o crescente consumo do produto, é um investimento lucrativo, mas que contém alguns riscos como custos do produto e variação da demanda.

\section{REFERÊNCIAS}

ALMEIDA, C. et al. Aspectos gerais da aplicação de sistemas de informação na agropecuária. Revista Eletrônica de Sistemas de Informação, Edição 8, n. 2. Volta Redonda, 2006.

FACHINI, A. Cenários da Tecnologia para 2014. Jornal do Brasil, São Paulo, 31 dez. 2013. Disponível em: <http://www.jb.com.br/sociedadeaberta/noticias/2013/12/31/cenarios-datecnologia-para-2014/>. Acesso em 16 de maio de 2014.

OLIVEIRA, M. A Avaliação Econômica Financeira de Investimentos Sob Condição de Incerteza: Uma Comparação Entre o Método de Monte Carlo e o VPL Fuzzy. Dissertação (Mestrado em Engenharia de Produção) Universidade de São Paulo, São Carlos, 2008.

PESSOA, M. F. Avaliação nutricional de diferentes rações comerciais em coelhos em crescimento. Dissertação (Mestrado em Zootecnia) Universidade Federal Rural do Rio de Janeiro, Seropédica, 2003. 